\title{
CONOCIMIENTOS Y PRÁCTICAS ANCESTRALES Y TRADICIONALES QUE FORTALECEN LA SUSTENTABILIDAD DE LOS SISTEMAS HORTÍCOLAS DE LA PARROQUIA DE SAN JOAQUÍN
}

\author{
ANCESTRAL KNOWLEDGE AND TRADITIONAL PRACTICES WHICH \\ STRENGTHEN THE SUSTAINABILITY OF HORTICULTURAL SYSTEMS IN SAN \\ JOAQUIN PARISH
}

\section{Juan Loyola I.}

Universidad Politécnica Salesiana, Campus el Vecino, Cuenca, Ecuador.

Autor para correspondencia: jloyola@ups.edu.ec

Manuscrito recibido el 25 de febrero de 2016. Aceptado, tras revisión, el 10 de octubre de 2016.

\begin{abstract}
Resumen
Esta investigación demuestra el papel determinante del conocimiento ancestral y tradicional en la sustentabilidad de los sistemas hortícolas familiares campesinos. Se desarrolló un estudio de caso con agricultores de la parroquia de San Joaquín del Cantón Cuenca, Ecuador. Se reconoce la estabilidad de la productividad de los sistemas hortícolas a través del tiempo. Se utilizó el Marco para la Evaluación de Sistemas de Manejo de Recursos Naturales incorporando Indicadores de Sustentabilidad (MESMIS). Se compararon las prácticas productivas de una finca convencional con nueve fincas caracterizadas como familiares campesinas con empleo de prácticas agroecológicas. Se identificaron puntos críticos de los sistemas hortícolas relacionados con la sustentabilidad y su combinación con la intensificación de la producción. Se establecieron relaciones entre las prácticas ancestrales y tradicionales y el desempeño de los indicadores evaluados. Se determinó entre el $60 \%(3 / 5)$ y el $78 \%(3,9 / 5)$ de las fincas satisfacen los umbrales de los indicadores ambientales. Sin embargo, los indicadores sociales y económicos tuvieron un rango mayor entre el $64 \%$ $(3,2 / 5)$ y el $98 \%(4,9 / 5)$ de satisfacción y entre el $60 \%(3 / 5)$ y $78 \%(3,9 / 5)$ respectivamente. Así se concluyó que los sistemas hortícolas cuentan con un alto grado de sustentabilidad, demostrado por un alto desempeño de los indicadores ambientales, económicos y sociales. Las prácticas ancestrales y tradicionales que desarrollan los campesinos, como: la asociación y rotación generan una amplia biodiversidad en la producción del sistema, tiene influencia directa en la generación de beneficios económicos y mejoramiento de la calidad de vida de las familias campesinas, son los elementos más relevantes.
\end{abstract}

Palabras claves: agroecología, agricultura tradicional, diversidad, horticultura, sustentabilidad. 


\begin{abstract}
This paper aims to show the determinant role of ancestral and traditional knowledge to achieve sustainability of horticultural systems. A study case with peasants of Parroquia San Joaquín, Cantón Cuenca, was developed. A key element was to acknowledge the stability of productivity in such horticultural systems across time. For this purpose, we implemented The Framework for the Evaluation of Natural Resource Management Systems Incorporating Sustainability Indicators (MESMIS). Production practices of a conventional farm and nine farms characterized as agroecological were compared. Critical points of horticultural systems related to sustainability and the relationship with production intensification were identified. Results achieved show that between $60 \%(3 / 5)$ and $78 \%(3,9 / 5)$ of the farms satisfy the environmental indicators thresholds. However, the social or economic indicators had a greater range between $64 \%(3,2 / 5)$ and $98 \%(4,9 / 5)$ fulfillment and $60 \%(3 / 5)$ and $78 \%(3,9 / 5)$ respectively. The study concludes that the horticultural systems have a high degree of sustainability, demonstrated by the high performance of environmental, economic and social indicators. The ancestral and traditional practices developed by peasant farmers such as association for production and commercialization, with a direct influence in the generation of economic benefits and improvements in life quality of peasant family, are the most relevant elements.
\end{abstract}

Keywords: agroecology, traditional agricultura, diversity, horticulture, sustainability.

Forma sugerida de citar: Loyola, J.. 2016. Conocimientos y prácticas ancestrales y tradicionales que fortalecen la sustentabilidad de los sistemas hortícolas de la parroquia de San Joaquín. La Granja: Revista de Ciencias de la Vida. Vol. 24(2):29-42. ISSN: 1390-3799. 


\section{Introducción}

\subsection{Situación actual de la agricultura fami- liar en el Ecuador}

El Estado Ecuatoriano ha considerado históricamente el sector campesino como ineficiente y atrasado puesto que emplea demasiada mano de obra, en sistemas generalmente de subsistencia que no genera economía de escala para la exportación. Sin embargo, es un hecho bien conocido que la agricultura campesina abastece alrededor del $70 \%$ de la demanda de alimentos de toda la América Latina. No obstante, el gasto en agricultura campesina es escaso, no solamente en tecnología, sino también en mejores condiciones de vida para los campesinos. Los mayores recursos financieros son comúnmente empleados en la agricultura para la exportación, lo cual perjudica directamente la producción doméstica. En general, según el Banco Central del Ecuador, la contribución al PIB de la agricultura desde el año 2000 hasta el 2012 ha decrecido desde 13,4 a $7,9 \%$ con una fuerte tendencia a la concentración de la tierra. Sin embargo, la agricultura juega un papel importante en la generación de empleos que contribuyen a la activación de la economía local.

Se estima que en Ecuador hay unos 250000 fincas familiares ( $30 \%$ del total) y que alrededor de tres millones de personas viven de la agricultura familiar (Martínez, 2013). Abarca unas 4 millones de hectáreas (33\% del total) y aportan el $60 \%$ de la canasta básica (Telégrafo, 2013). De ellos el 61,6\% tienen una agricultura familiar de subsistencia (AFS), 37\% tienen una agricultura familiar en transición (AFT), y el 1,4\% una agricultura familiar consolidada (AFC) (Secretaría General de la Comunidad Andina (2011) tomado de: FAO/BID (2007)).

La agricultura familiar como actividad agrícola y social considera el cultivo de una superficie de tierra para la producción de alimentos con destino al consumo interno y los excedentes para la comercialización. Los integrantes de la familia participan en el trabajo, la toma de decisiones en estos aspectos. La agricultura familiar se sustenta en procesos culturales a nivel de finca, localidad o territorio, preserva tradiciones y conocimientos, no solamente respecto a la producción de alimentos, sino también en las prácticas ecológicas respetuosas de la naturaleza. Por ello, se reconoce ampliamente el papel de salvaguarda de la naturaleza que cumple la agricultura tradicional campesina.
La producción familiar contribuye a la seguridad y soberanía alimentaria en los países Andinos, mientras que favorece la conservación de la biodiversidad y activa las economías rurales y preserva la cultura campesina. En la relación directa y especial que establece la agricultura campesina con la naturaleza, la cultura, la economía y la sociedad, emerge un alto potencial para el desarrollo sustentable de la producción de alimentos. Tal sustentabilidad parece ser posible de alcanzar a partir de un balance armónico entre los procesos ecológicos y los socio-económicos. Sin embargo, es frecuente que no se reconozcan estas virtudes de la agricultura campesina familiar, con lo cual se margina el potencial que esta tiene para contribuir al desarrollo social más amplio del país.

Todos los rasgos mencionados anteriormente, junto a otros muchos le confieren un carácter multifuncional a la agricultura familiar, que es capaz de proveer de bienes y servicios al entorno natural y ecológico, cuando se trabaja a una escala compleja y dinámica de pequeña producción. Por ser diversificada y manejada con un alto nivel de detalle, tiene la capacidad de ser energéticamente eficiente, resiliente a diversos fenómenos naturales extremos y desbalances socio-económicos, así como de mitigar y adaptarse al cambio climático.

\subsection{La producción hortícola}

Los modos de producción agrícola y hortícola en particular, propios de la parroquia San Joaquín del cantón Cuenca, son el resultado de una evolución eco-social o socio-ecológica. Esta evolución se refiere a la mezcla de las tradiciones y métodos de cultivo y manejo del suelo de pobladores indígenas de la zona con aquellos portados por los conquistadores españoles en su colonización del territorio. Sin embargo, en lo que se refiere a los hábitos alimenticios, es más reciente que se haya incorporado el consumo de hortalizas en la dieta diaria (hacia la década de los 90 del siglo pasado). Este hecho ha ofrecido una oportunidad a los agricultores para aumentar la oferta de productos y una mayor seguridad de comercialización en los mercados locales. La posibilidad de realizar de tres a cuatro cosechas de hortalizas, debido a las favorables condiciones climáticas, el ciclo corto de la mayoría de ellas y los sistemas de cultivo empleados, ha permitido fortalecer las relaciones con los mercados, satisfaciendo la demanda local y a su vez ha permitido la activación de 
la agricultura campesina dedicada a la producción hortícola.

La horticultura ecuatoriana se ha desarrollado en la región sierra debido a las condiciones de suelo, ambientales y socioeconómicas. El sistema de producción hortícola es familiar ya que todos aportan en el proceso productivo a través de la mano de obra de cada uno de los miembros que integran la familia. Una parte de la producción que se obtiene está dirigida al autoconsumo y sus excedentes les permite comercializar en los mercados.

La horticultura en el Ecuador se ha desarrollado a partir del proceso de "modernización" agraria, basada en el enfoque de la agricultura científica (convencional) que les mantiene a los agricultores medianos y pequeños atados a los paquetes tecnológicos preestablecidos (semillas, abonos, fertilizantes, fungicidas, herbicidas, etc.). La reactivación de la economía campesina dentro del país se basó a partir de la horticultura, teniendo como dificultad esta actividad agrícola la dependencia total de la importación de semillas.

\subsection{La agroecología}

Este enfoque ambientalmente productivo, socialmente equilibrado y económicamente viable, aplica los principios y conceptos ecológicos al diseño y manejo de los agroecosistemas (Altieri, 1995; Gliessman, 2002).

La agroecología como disciplina que permite el entendimiento de los elementos y funcionalidad de las prácticas de la agricultura ancestral y tradicional, contribuye a concebir la sostenibilidad en la agricultura (Estermann, 1998), y así logra una transformación social, ambiental y económica, que sienta las bases de un uso equilibrado y a la vez productivo de los agroecosistemas locales.

Los sistemas ancestrales y tradicionales se encuentran vinculados a la organización familiar y social, valores, tecnología y ambiente, logrando una diversidad ecológica, cultural y socioeconómica como estrategias de sobrevivencia desarrollada por los agricultores (Bernstein, 2012).

En el tiempo la horticultura familiar ha venido desarrollando sistemas productivos menos dependientes de insumos excepto de las semillas. Los sistemas agrícolas de la Parroquia de San Joaquín, posee áreas bajo riego y son apropiadas para el cultivo de una amplia gama de hortalizas durante todo el año, donde se realiza una producción intensiva en pequeñas áreas como una actividad de la agricultura familiar.

$\mathrm{Al}$ ser sistemas intensivos, se produce un deterioro ambiental. Los horticultores han desarrollado prácticas apropiadas basadas en un buen uso de los recursos prediales (el suelo, agua, biodiversidad, etc.), a través de las prácticas que se encuentran dentro del itinerario técnico, basadas en los conocimientos ancestrales y tradicionales, combinados con el conocimiento científico, lo que favorece que estas fincas hortícolas hayan perdurado en el tiempo.

$\mathrm{Al}$ establecer las diversas prácticas del itinerario técnico y la incidencia que tienen en su conjunto dentro de los sistemas hortícolas, se conoce y establece cuáles de estas prácticas favorece un uso más eficiente de la energía y cuáles deben incorporarse para llegar a un sistema agroecológico.

\subsection{Objetivo}

Evaluar la influencia de los conocimientos y prácticas ancestrales y tradicionales de las familias campesinas en la productividad y sustentabilidad de la producción hortícola en la Parroquia de San Joaquín, Cuenca, Ecuador.

\section{Materiales y Métodos}

Se seleccionaron 10 fincas hortícolas familiares de la parroquia de San Joaquín, Cantón Cuenca, Ecuador. Se identificaron puntos críticos para la evaluación de las fincas, considerando tres dimensiones básicas de sistemas agrícolas sustentables: estabilidad ecológica, rentabilidad económica y aceptabilidad social. Se construyó un conjunto de indicadores cualitativos y cuantitativos que sirvieran para caracterizar y evaluar los sistemas de manejo y las condiciones socioeconómicas propias de cada uno.

Para evaluar la influencia de las prácticas campesinas en la producción hortícola, se empleó la metodología de la Investigación Participativa Revalorizadora (IPR), la cual tiene el rol de generar conocimiento y/o recrear saberes locales desde la perspectiva de los actores sociales, permite comprender la realidad (vida material, vidas social y vida espiritual), en un determinado contexto espacio temporal (Delgado, 2010).

La investigación permitió entender y analizar de manera objetiva la realidad de cada horticultor, en 
base al conocimiento local y la participación activa en todo el proceso a través del diálogo intra e intercultural y multidisciplinar, generando un ambiente de confianza desde la conversación (el relato). Se realizó diez entrevistas semiestructuradas, 40 conversaciones informales, un grupo de discusión y 40 visitas de campo, a través del siguiente enfoque metodológico:

- Trabajo de campo, se inició con un acercamiento a los agricultores para presentar y recibir la aprobación de la investigación.

- Técnicas de investigación, para la recolección de la información cualitativa y cuantitativa que involucran activamente a los agricultores como protagonistas de la información son: diez entrevistas, diez estudios de caso, dos talleres de discusión.

- Validación del proceso investigativo, obtenidos los datos de sustentabilidad se puso a consideración de los horticultores para complementar la información cuando ellos creyeron necesario y su posterior validación, confirmando así la veracidad de la información y los resultados.

Para la identificación de puntos críticos y evaluación de indicadores de sustentabilidad, se empleó el Marco para la Evaluación de Sistemas de Manejo de Recursos Naturales incorporando Indicadores de Sustentabilidad (MESMIS) (Astier et al., 2008), que permitió la comparación transversal de un sistema convencional y sistemas agroecológicos (Masera, 2000).

\subsection{Selección de estudios de caso}

Se seleccionó la finca del señor Francisco Villasis que ha mantenido durante al menos los últimos 5 años un manejo convencional del sistema productivo, quiere decir, uso de fertilizantes químicos, herbicidas y plaguicidas, etc., otras nueve familias seleccionadas para el estudio desarrollan de manera regular innovaciones tecnológicas de acuerdo a sus necesidades y posibilidades para reducir la dependencia de insumos, teniendo el sistema de la señora Dolores Duchitanga presenta una mayor práctica de agroecología dentro de su sistema.
La Figura 1 muestra un modelo del sistema de conocimiento local de los sistemas hortícolas de la parroquia de San Joaquín, adaptado a su realidad cultural y su relación con la naturaleza, definiendo el itinerario técnico de sus componentes productivos (Luger et al., 2003). Para un análisis holístico de los sistemas hortícolas se requirió identificar la mayor cantidad posible de componentes e interrelaciones que intervienen en el proceso.

La evaluación de la sostenibilidad partió de la asociación de los atributos conectados con criterios de diagnóstico a través de un análisis más detallado que vinculan a los indicadores permitiendo una evaluación efectiva (Masera, 2000). Los indicadores establecidos son específicos para el estudio que son fáciles de observar, medir y entender.

Los criterios para los indicadores fueron: sean fáciles de obtener, de interpretar y nos brinden la información necesaria para establecer tendencias de las fincas. Los indicadores estuvieron fortalecidos por subindicadores y variables seleccionadas y cuantificadas que integran con facilidad la capacidad de evaluar las prácticas de manejo sobre el sistema hortícola.

La Tabla 1 muestra los indicadores seleccionados, los cuales fueron estandarizados en una escala positiva de 1 a 5, así como ponderados de acuerdo a su importancia y confiabilidad. Los datos para la construcción de dichos indicadores se obtuvieron a través de entrevista a los productores y observaciones en las parcelas.

\section{Resultados y discusión}

\subsection{Dimensión ambiental}

En la Figura 2 se procede a analizar e integrar los resultados obtenidos, de acuerdo a los 6 indicadores:

\section{A1. Diversidad de cultivo, asociación y rotación, de} las diez fincas el promedio es de 3,2. Alcanzando el sistema agroecológico el nivel máximo de 5 y el sistema convencional de 3 . Estableciendo que a pesar de la influencia de la agricultura convencional se practica y tiene mucha incidencia del conocimiento ancestral y tradicional, en especial al asociación, rotación y abonadura orgánica. 
Figura 1. Componentes de los agroecosistema hortícolas de la parroquia de San Joaquín del cantón Cuenca-Ecuador. 
Tabla 1. Puntos críticos, criterios de diagnóstico e indicadores para evaluar la sustentabilidad de las fincas hortícola.

\begin{tabular}{|c|c|c|c|}
\hline Atributos & Criterio de diagnóstico & Indicador estratégico & Área evaluada \\
\hline Productividad & Eficiencia & $\begin{array}{l}\text { 1. Dependencia de insumos externos } \\
\text { 2. Ingreso por producción }\end{array}$ & $\begin{array}{c}\mathrm{A} / \mathrm{E} \\
\mathrm{E}\end{array}$ \\
\hline \multirow{4}{*}{$\begin{array}{l}\text { Estabilidad, } \\
\text { resiliencia, } \\
\text { confiabilidad }\end{array}$} & Diversidad & $\begin{array}{l}\text { 3. Diversidad de cultivo, asociación y rotación } \\
\text { 4. Numero de cultivos en producción y destino }\end{array}$ & $\begin{array}{l}\text { A } \\
\mathrm{E}\end{array}$ \\
\hline & Conservación de recurso & 5. Características biofísicas del agua y suelo & A \\
\hline & Calidad de vida & 6. Bienestar familiar & $\mathrm{S}$ \\
\hline & Fragilidad del sistema & 7. Alteraciones climáticas & A \\
\hline Adaptabilidad & Capacidad de cambio e innovación & $\begin{array}{l}\text { 8. Acceso a innovación agrícola } \\
\text { 9. Generación de prácticas y conocimientos }\end{array}$ & $\begin{array}{c}\mathrm{S} \\
\mathrm{S} / \mathrm{A}\end{array}$ \\
\hline Equidad & Distribución de costos & $\begin{array}{l}\text { 10. Ingresos netos por producción } \\
\text { 11. Número de beneficiarios }\end{array}$ & $\begin{array}{l}\mathrm{E} \\
\mathrm{S}\end{array}$ \\
\hline \multirow{4}{*}{ Autogestión } & Organización & $\begin{array}{l}\text { 12. Manejo de registros de producción y econó- } \\
\text { micos } \\
\text { 13. Toma de decisiones } \\
\text { 14. Mercado local y provincial }\end{array}$ & $\begin{array}{l}\text { A } \\
\text { S } \\
\text { S }\end{array}$ \\
\hline & Autosuficiencia & 15. Producción continua & A \\
\hline & Control & 16. Uso de conocimientos y habilidades locales & $\mathrm{A} / \mathrm{S}$ \\
\hline & Participación & $\begin{array}{l}\text { 17. Mano de obra local y externa } \\
\text { 18. Equidad económica }\end{array}$ & $\begin{array}{l}\mathrm{S} \\
\mathrm{E}\end{array}$ \\
\hline Productividad & Eficiencia & $\begin{array}{l}\text { 19. Dependencia de insumos externos } \\
\text { 20. Ingreso por producción }\end{array}$ & $\begin{array}{c}\mathrm{A} / \mathrm{E} \\
\mathrm{E}\end{array}$ \\
\hline \multirow{4}{*}{$\begin{array}{l}\text { Estabilidad, } \\
\text { resiliencia, } \\
\text { confiabilidad }\end{array}$} & Diversidad & $\begin{array}{l}\text { 21. Diversidad de cultivo, asociación y rotación } \\
22 \text {. Numero de cultivos en producción y destino }\end{array}$ & $\begin{array}{l}\mathrm{A} \\
\mathrm{E}\end{array}$ \\
\hline & Conservación de recurso & 23. Características biofísicas del agua y suelo & A \\
\hline & Calidad de vida & 24. Bienestar familiar & $\mathrm{S}$ \\
\hline & Fragilidad del sistema & 25. Alteraciones climáticas & A \\
\hline Adaptabilidad & $\begin{array}{l}\text { Capacidad de cambio e } \\
\text { innovación }\end{array}$ & $\begin{array}{l}\text { 26. Acceso a innovación agrícola } \\
\text { 27. Generación de prácticas y conocimientos }\end{array}$ & $\begin{array}{c}\mathrm{S} \\
\mathrm{S} / \mathrm{A}\end{array}$ \\
\hline Equidad & Distribución de costos & $\begin{array}{l}\text { 28. Ingresos netos por producción } \\
29 . \text { Número de beneficiarios }\end{array}$ & $\begin{array}{l}\mathrm{E} \\
\mathrm{S}\end{array}$ \\
\hline \multirow{4}{*}{ Autogestión } & Organización & $\begin{array}{l}\text { 30. Manejo de registros de producción y econó- } \\
\text { micos } \\
\text { 31. Toma de decisiones } \\
\text { 32. Mercado local y provincial }\end{array}$ & $\begin{array}{l}\text { A } \\
\text { S } \\
\text { S }\end{array}$ \\
\hline & Autosuficiencia & 33. Producción continua & A \\
\hline & Control & 34. Uso de conocimientos y habilidades locales & $\mathrm{A} / \mathrm{S}$ \\
\hline & Participación & $\begin{array}{l}\text { 35. Mano de obra local y externa } \\
\text { 36. Equidad económica }\end{array}$ & $\begin{array}{l}S \\
E\end{array}$ \\
\hline
\end{tabular}

(Nota: A- ambiental, E- económica, S- social). 



Figura 2. Diagrama en tela de araña representando la dimensión ambiental de las fincas hortícolas familiares estudiadas de la parroquia de San Joaquín del cantón Cuenca-Ecuador.
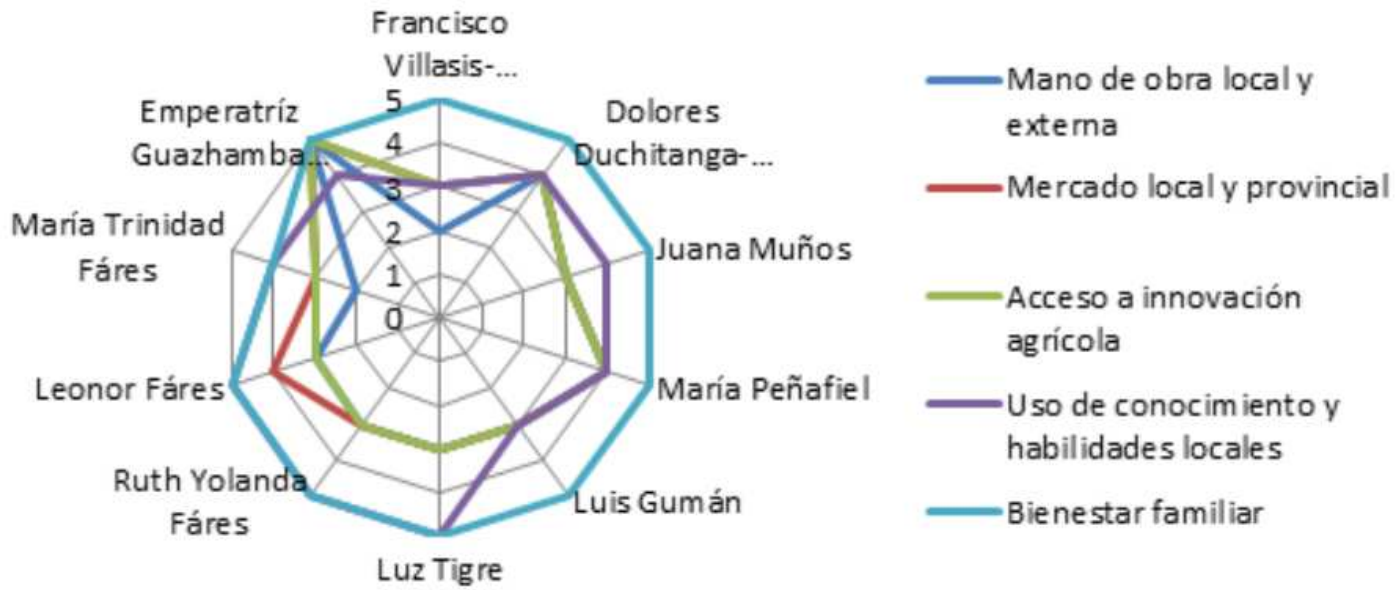

Figura 3. Diagrama en tela de araña representando la dimensión social de las fincas hortícolas familiares estudiadas de la parroquia de San Joaquín del cantón Cuenca-Ecuador. 
A2. La dependencia de insumos externos, el promedio de las fincas estudiadas es de 3,3 alcanzando 2 el sistema agroecológico y el sistema convencional llega a 3 . El sistema convencional requiere de energía agroquímica (herbicidas, abonos, insecticidas, fungicidas, etc.) y semillas, haciendo que esto genere una diferencia significativa entre los demás sistemas debido a que los sistemas agroecológicos utilizan con menor frecuencia abono orgánico, lo que hace que disminuya en tiempo el volumen, requiriendo solamente semilla o plántulas para cada ciclo productivo.

A3. Manejo de registros de producción y económicos, alcanza un promedio de 3 de las diez fincas estudiadas. La finca agroecológica y la finca convencional obtuvieron 2 dentro de la escala. Las familias de cada uno de los sistemas productivos saben con exactitud las áreas productivas, las especies cultivadas o a cultivar, los gastos en cada cultivo, época de cosecha e ingreso que obtienen, evidenciando que no es parte de la cultura el llevar registro de sus actividades como medio de verificación.

A4. Características biofísicas del agua y suelo, se establece un promedio de 3,8 en las fincas estudiadas. La finca agroecológica tiene un indicador de 4,5 y la finca convencional de 4 . Los agricultores dentro de los sistemas productivos son conscientes del cuidado de los recursos, en el caso de la finca convencional el agricultor por sus años de experiencia usa los productos agroquímicos bajo las recomendaciones establecidas porque sabe que le lleva al ahorro de dinero. También se podría establecer que la contaminación no es muy alta debido a que los sistemas de riego se encuentra entubado.

A5. Uso de conocimientos y habilidades locales, se establece un promedio de 3,7 donde la finca agroecológica tiene 4 y la finca convencional alcanza 3. Los conocimientos ancestrales y tradicionales no desaparecen a pesar que se trabaja con cultivos introducidos, potenciando así sus sistemas productivos.

A6. Producción continúa, los sistemas hortícolas alcanzan un promedio de 3,9. El sistema agroecológico logra un valor de 5 y el sistema convencional alcanza 3. Estos datos representan que los horticultores optimizan el recurso suelo a través de la asociación y rotación, son conocimientos y prácticas ancestrales y tradicionales. También es debido al conocimiento del desenvolvimiento del mercado hortícola.

\subsection{Dimensión social}

El grado de satisfacción de los aspectos sociales intrafamiliares, en los cuales los horticultores tienen asegurado la alimentación, sus necesidades básicas (vivienda, educación, salud, etc.), la relación familiar con otras familias o miembros de la comunidad mediante la actividad agrícola a través de la generación de empleo, llevan una redistribución de la riqueza, como se observa en la Figura 3.

S1. Mano de obra local y externa, se establece un promedio 3,2 en las diez fincas estudiadas. En el sistema agroecológico alcanza una escala de 4 y el sistema sistema convencional de 2. Evidenciando que el sistema agroecológico requiere significativamente de más mano de obra en sus actividades productivas que el del sistema convencional, lo cual estableció una alta participación de la mano de obra familiar y el aporte de la mano de obra externa.

S2. Mercado local y provincial, se logra un promedio de 3,5 en los sistemas estudiados, el sistema agroecológico alcanza un indicador de 4 y el sistema convencional de 3. En el que hay una diferencia significativa entre los dos sistemas que nos lleva a establecer que el sistema agroecológico presenta diversidad de productos y continuidad en la producción para la venta.

S3. Acceso a innovación agrícola, alcanza un promedio de 3,4 de las diez fincas estudiadas. El sistema agroecológico llega a 4 y la finca convencional a 3, se estableció por lo tanto que la familia del sistema agroecológico aprovecha eventos de capacitación que logran un mayor conocimiento de prácticas de protección y productivas.

\section{S4. Uso de conocimiento y habilidades locales,}

alcanza un valor promedio de 4,1. El sistema agroecológico alcanza un valor de 4 y el sistema convencional 3. Se puede afirmar que la finca agroecológica desarrolla con mayor 


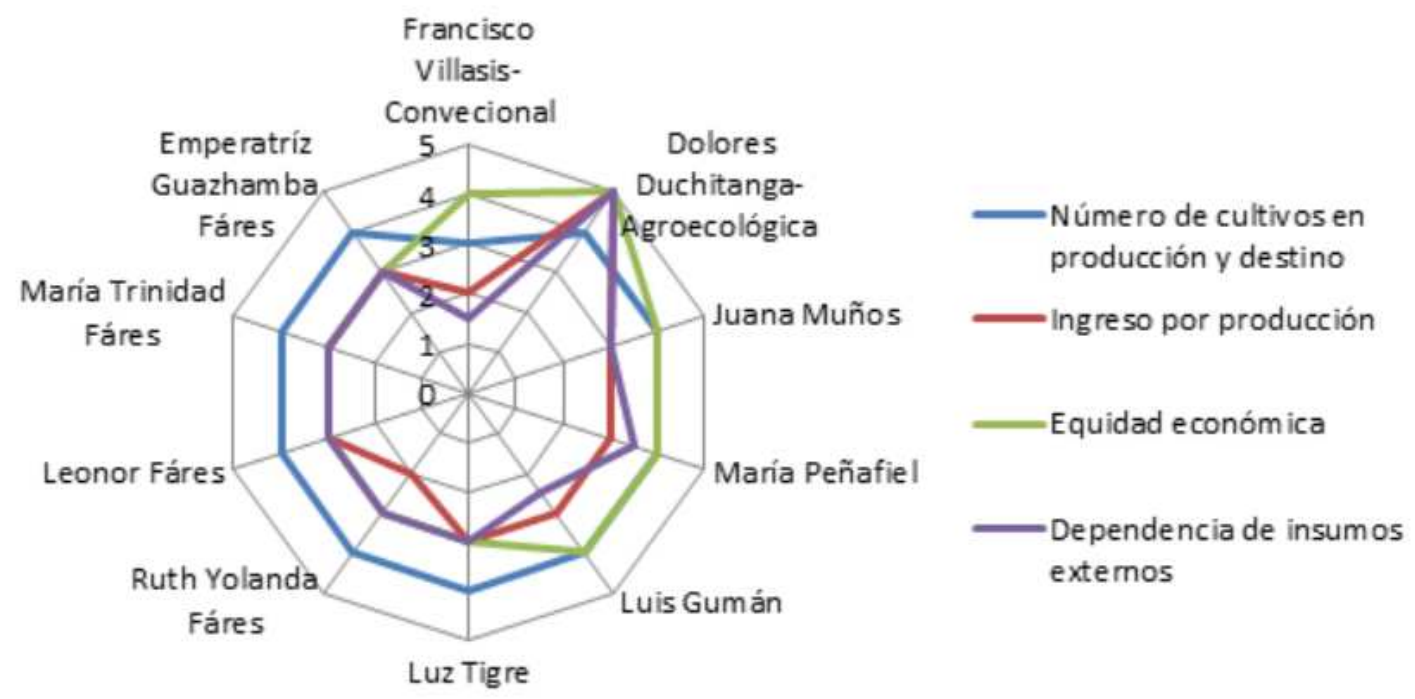

Figura 4. Diagrama en tela de araña representando la dimensión económica de las fincas hortícolas familiares estudiadas de la parroquia de San Joaquín del cantón Cuenca-Ecuador.

intensidad el conocimiento, prácticas y habilidades (ancestrales y tradicionales) locales dentro del sistema hortícola.

S5. Bienestar familiar, en las fincas estudiadas se cuenta con un promedio de 4,9. En donde el sistema agroecológico y el sistema convencional tienen un valor de 5 . Se estableció que la satisfacción de los miembros de las familias que son parte de los sistemas tiene un alto grado de satisfacción, es decir; tienen aseguradas sus necesidades básicas, alcanzando en gran parte contar con ahorro, llevándoles a estar satisfechos con sus sistemas productivos.

\subsection{Dimensión económica}

Para evaluar si los sistemas hortícolas son económicamente viables se estudiaron los siguientes indicadores de los cuales se llegó a los consiguientes resultados (Figura 4):

E1. Número de cultivos en producción y destino, el promedio de este indicador de las fincas estudiadas es de 3,9. La finca agroecológica al- canza un valor de 4 y la finca convencional de 3. Se estableció, por lo tanto los sistemas hortícolas cuentan con un alto número de cultivos en sus áreas (asociación), donde se establece la maximización del recurso suelo demostrando una mayor capacidad productiva en el sistema agroecológico a través de la diversificación.

E2. Ingreso por producción, en las fincas investigadas el promedio es de 3, la finca agroecológica logra el valor máximo de 5 y la finca convencional un valor de 2. En donde se establece que las fincas agroecológicas han llegado a su máxima capacidad productiva a través de la aplicación y desarrollo de las prácticas de asociación y rotación de cultivos.

E3. Equidad económica, dentro de este indicador los sistemas productivos alcanzan un promedio de 3,6. La finca agroecológica cumple con el máximo valor de 5 y finca convencional obtiene 4. Estableciendo que los sistemas son trabajados con decisiones y participación del grupo familiar. 


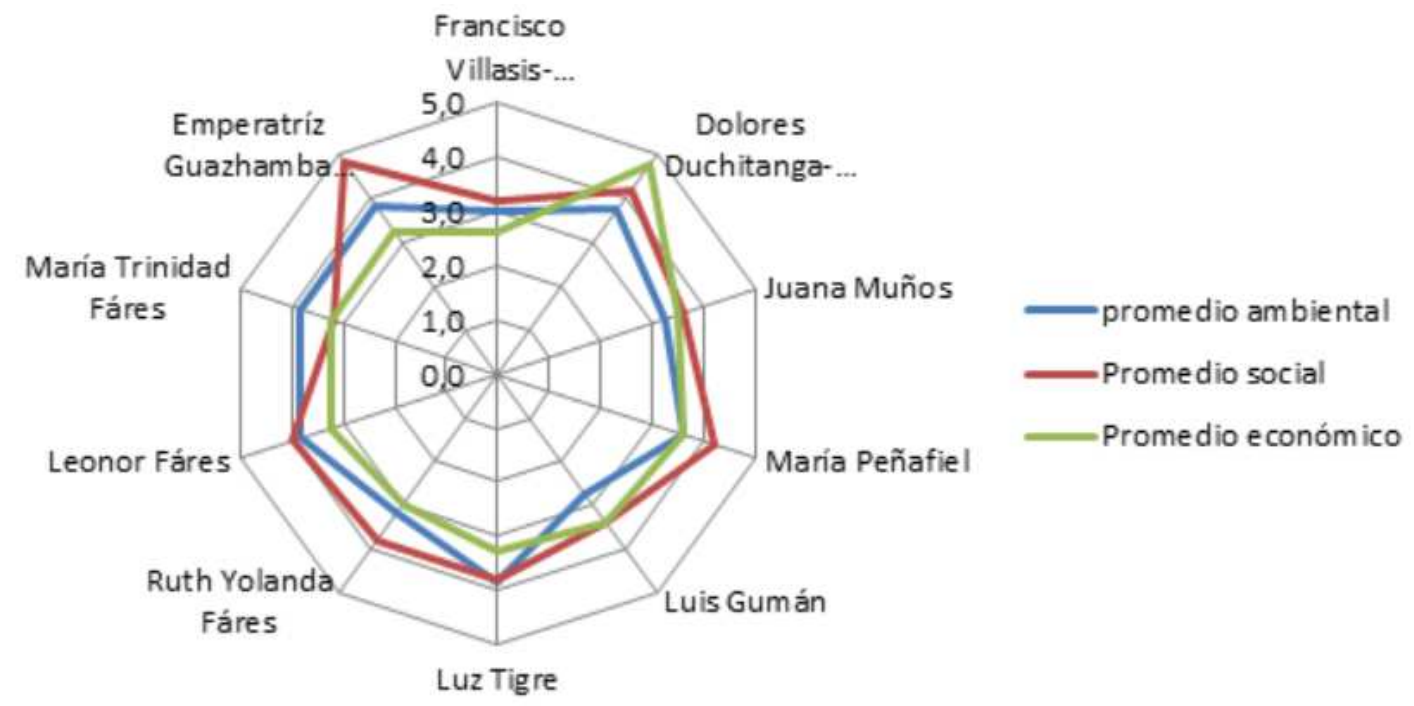

Figura 5. Diagrama en tela de araña representando el promedio de la dimensión ambiental, social y económica de las fincas hortícolas familiares estudiadas de la parroquia de San Joaquín del cantón Cuenca-Ecuador.

E4. Dependencia de insumos externos, se determinó que esta variable alcanza un promedio de 3,05. El valor del sistema agroecológico es de 5 y el sistema convencional de 1,5. Estableciendo que los sistemas agroecológicos requieren en mayor volumen el abono orgánico (pollinaza o gallinaza) y semillas, los sistemas convencionales el volumen es menor ya que requieren menos cantidad y sus siembras no son tan intensivas como los sistemas agroecológicos.

\subsection{Integración de las dimensiones ambien- tales, sociales y económicas}

Como se observa en la Figura 5, en la cual se integran la dimensión social, ambiental y económica, se estableció que las fincas hortícolas se encuentran por encima del promedio medio de la escala establecida en el estudio (escala de 1 como mínimo y 5 como máximo). La diferencia de los sistemas productivos hortícolas están de acuerdo a los objetivos que persigue cada familia y la disponibilidad del recurso suelo (entre $1.200 \mathrm{~m}^{2}$ hasta $10.250 \mathrm{~m}^{2}$ como máxi$\mathrm{mo})$, son elementos base que definen a cada una de las fincas. Luego de este análisis los agricultores establecen la disponibilidad de mano de obra familiar y externa con la que cuentan, quedando por último las necesidades de insumos establecidas por los horticultores.
Desde la dimensión ambiental, las prácticas de manejo utilizadas en un principio en la producción hortícola mostraron un impacto adverso, pero actualmente estas prácticas están siendo llevadas a base de un conocimiento ancestral y tradicional que favorece la producción que mantienen y mejoran los recursos internos como externos a la finca, alcanzando un valor promedio de 3,48 de las diez fincas, la finca agroecológica obtiene 3,75 y la finca convencional 3, estos resultados evidencian la capacidad de los horticultores a modificar y acomodar a través de los Saberes Ancestrales y Tradicionales la producción hortícola a un modelo propio y ambientalmente favorable.

En cuanto a la dimensión social existe una fortaleza ya que toda la familia participa en la toma de decisiones, en la organización productiva y comercialización. El promedio de las diez fincas es de 3,82; lo cual correspondió para la finca agroecológica 4,2 y para la finca convencional 3,2. Esto evidenció que el sistema agroecológico hay una mayor integración y cohesión familiar en todas las actividades del sistema, igualmente es un principio socio cultural andino que se encuentra dentro de los Saberes Ancestrales y Tradicionales.

En la dimensión económica se observó una rentabilidad económica media alta, debido a que los productores dirigen el 95,34\% del total a la venta. También han logrado una alta productividad debi- 


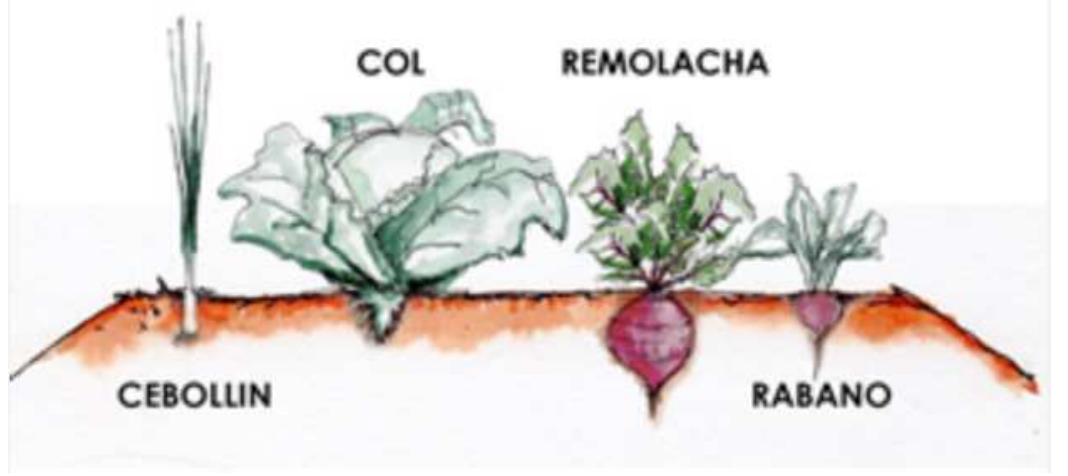

Figura 6. Arreglo hortícola en los cultivos.

do que han desarrollado ajustes al modelo convencional, reemplazando insumos inorgánicos por insumos orgánicos y diversificando los cultivos (asociación y rotación), consiguiendo mayor productividad por área, mejor calidad y en diferente tiempo, alcanzando un promedio las diez fincas de 3,48 que es un valor significativo, el sistema agroecológico alcanza 4,75 y el sistema convencional de 2,63. Se evidenció que el sistema agroecológico aplica y desarrolla los Saberes Ancestrales y Tradicionales con mayor intensidad en todo el sistema productivo.

Los sistemas hortícolas se encuentran acompañados de un alto riesgo económico debido a la dependencia de insumos importados como la semilla y agroquímicos, que provocan inestabilidad a los sistemas por esta dependencia.

\section{Conclusiones}

Al evaluar la sustentabilidad de los sistemas hortícola a través del uso de indicadores a los agroecosistemas de pequeños productores hortícolas, se determinó que los valores son un instrumento válido para conocer el nivel o grado de bienestar familiar a través de la dimensión ambiental, social y económica.

Dentro del estudio, a pesar de la aparente variabilidad de las fincas hortícolas se estableció una alta similitud en las diferentes dimensiones. En promedio, tanto la finca convencional como las nueve fincas agroecológicas alcanzaron un valor superior al valor medio de la escala, encontró variaciones muy cortas entre los valores de cada indicador de las tres dimensiones estudiadas, a partir de este estudio se demuestra que los conocimientos, prácticas y conductas sociales deben ser revalorizados por que pueden reproducir en otras zonas rurales (Toledo, 1998).

El manejo de los sistemas hortícolas satisface en gran medida al objetivo económico de los agricultores, lo cual evidencia que son sistemas llevados con base al Conocimiento y Saberes Ancestrales y Tradicionales prioriza elementos ecológicos y socioculturales (Costanza y Daly, 1992). El Conocimiento y Saberes Ancestrales y Tradicionales se fundamenta en el uso de la biodiversidad a través de la asociación y rotación en los sistemas productivos, es una expresión de la implementación de las estrategias del uso múltiple, que responde a una racionalidad tanto ecológica, sociocultural y en la actualidad a la económica, reafirmando lo que manifiesta Romero-Arenas (2010). Estas prácticas según Vázquez (2008) los sistemas hortícolas influyen favorablemente para disminuir la presencia y desarrollo de las plagas, favoreciendo ambiental y económicamente a los agricultores.

Los arreglos cronológicos que los horticultores establecen al cultivar dependen de la necesidad del mercado para realizar la siembra y el grado de traslape entre las diferentes especies (Figura 6), lo cual produce interacciones entre las diversas especies hortícolas que hacen que cada una tenga sus microrganismos asociados (Gliessman, 2002). Se logra así un balance a través de las interacciones, lo que proporciona estabilidad al sistema y un efecto directo en la reducción de los costos económicos y ambientales (Romero-Arenas, 2010). 
Según Guzmán, (2005), el costo de oportunidad de trabajo también es analizado por las familias y se confirma que a partir de él se realiza la distribución de la mano de obra familiar dentro de las actividades agropecuarias o extra agropecuarias, puesto que compara una hora de trabajo dentro de su finca, en relación con una hora de trabajo fuera de la explotación, estableciendo una lógica del costo de oportunidad de él y de su familia.

Los valores de sustentabilidad alcanzados confirman la existencia de cierta racionalidad de los sistemas productivos con menos dependencia de insumos que fomenta mecanismos de sustentabilidad como manifiesta Figueroa, (1991). Tales mecanismos se ponen en marcha en sistemas biodiversos y multifuncionales que son capaces de adaptarse a condiciones desfavorables. Se identificó que las prácticas agrícolas ancestrales y tradicionales se basan en la utilización de la diversidad como pilar fundamental del manejo, la fertilización orgánica y el cierre de los ciclos biogeoquímicos al interno de las fincas, además, se determinó que las prácticas favorecen la cobertura vegetal a través de asociaciones de cultivos y con los arvenses nativos y endémicos, y por último se puede considerar la labranza con bueyes según como manifiesta Ploeg, (1994).

Con estas prácticas medidas a través de los indicadores, se identificó que existe una alta interdependencia entre las tres dimensiones analizadas, lo cual evidencia que la sustentabilidad ecológica está claramente condicionada por los aspectos socioculturales (conocimiento y saberes ancestrales y tradicionales) y económicos. De igual manera se observó que estas prácticas genera una mayor estabilidad de los agroecosistemas y su autorregulación, provocando una mejor recuperación y conservación de los recursos, mejorando además, los aspectos económicos y sociales.

Los sistemas hortícolas engloban prácticas, técnicas, conocimientos y/o cosmovisiones que responden a fortalezas y deficiencias que favorecen o limitan la producción agrícola.

Estos conocimientos y saberes son generados a partir de una observación acuosa, sistémica y de convivencia con la naturaleza, que son transmitidos a la siguiente generación por tradición oral y trabajo de campo.

\section{Bibliografía}

Altieri, M. A. 1995. Agroecology: The science of sustainable agriculture, $2^{\text {nd }} \mathrm{Ed}$. Westview Press, Boulder, CO, USA.

Astier, M., O. Masera e Y. Galván-Miyoshi. 2008. Evaluación de sustentabilidad un enfoque dinámico y multidimensional. Catarroja: SEAE. páginas 26-47.

Bernstein, H. 2012. Dinámicas de clase y transformación agraria. Zacatecas: Miguel Ángel Porrúa. página 25.

Costanza, R. y H. E. Daly. 1992.Natural capital and sustainable development. Conservation Biology. 6:37-46. In Harte (1995) Ecology, sustainability and environment as capital. Ecological Economics. 15:157-164.

Delgado, R. 2010. Investigación Participativa Revalorizadora e Innovación Tecnológica. Enfoque Transdiciplinar en la Innovación de Saberes Agropecuarios. Ed. ARUCO-BioAndes. página 118.

Estermann, J. 1998. Filosofía andina: estudio intercultural de la sabiduría autóctona andina. Quito, Ecuador: Ediciones Abya-Yala. página 29.

Figueroa, Adolfo. 1991. "Desarrollo agrícola en la América Latina", en Oswaldo Sunker, El desarrollo desde adentro: un enfoque neoestructuralista para América latina. Fondo de Cultura Económica, México. páginas 361-382.

Gliessman, S. R. 2002. Agroecología: Processos ecológicos em agricultura sustentable, segunda Ed. Porto Alegre, Brasil: Editorial Universidade/ UFRGS. página 20.

Guzmán, E. Gómez. 2005. Resistencia, permanencia y cambio: estrategias campesinas de vida en el poniente de Morelos, $1^{\text {ra }}$ Ed. Cuernavaca, Morelos, México, D.F: Universidad Autónoma del Estado de Morelos; Plaza y Valdés. páginas 1-7.

La agricultura familiar, clave para asegurar la soberanía alimentaria. (13 de julio de 2014). El telégrafo. página 8.

La agricultura familiar representa el $70 \%$ de la producción. (21 de junio de 2013). El Telégrafo. página 8 . 
Luger, Anton, Dara Cisneros y Guenrij Silva. 2003. Aprendiendo de nuestra experiencia; manual de sistematización participativa. Quito, Ecuador: Abya-Yala. página 73.

Masera, O. Cerutti. 2000. Sustentabilidad y manejo de recursos naturales: el marco de evaluación MESMIS. México, D.F.: Pátzcuaro, Michoacán: Mundi-Prensa México; Grupo Interdisciplinario de Tecnología Rural Apropiada; Universidad Nacional Autónoma de México, Instituto de Ecología. ṕaginas 5-23.

Martínez, L. Valle. 2013. La Agricultura Familiar en El Ecuador. Serie Documentos de Trabajo N¹47. Grupo de Trabajo: Desarrollo con Cohesión Territorial. Programa Cohesión Territorial para el Desarrollo. Rimisp, Santiago, Chile.

Odum, H.T. 1983. Systems ecology: an introduction. New York: Wiley.

Ploeg, Jan Douwe van der. 1994. "Styles of Farming: An Introductory Note on Concepts and
Methodogy", en Jan Douwe van der Ploeg y Ann Long (eds.), Born from Within. Practice and Perspective of Endogenous Rural Development, Van Gorcum Publisher, Assen.

Romero-Arenas, M.A.D.-H. 2010. Manejo del maíz en el estado de Tlaxcala, México: entre lo convencional y lo agroecológico. Revista Latinoamericana de Recursos Naturales. páginas 6776, 237-247.

Sevilla, E. Guzmán. 2006. De la sociología rural a la agroecología. Barcelona: Icaria Editorial.

Toledo, Víctor Manuel. 1998. Campesinidad, agroindustrialidad, sostenibilidad. Los fundamentos ecológicos e históricos del desarrollo. Grupo Interamericano para el Desarrollo Sostenible de la Agricultura y los Recursos Naturales, Cuadernos de trabajo 3, México.

Vázquez, L. 2008. Manejo integrado de plagasPreguntas y respuestas para técnicos y agricultores. Habana-Cuba: Editorial CientíficoTécnico. 